\title{
Namenregister Nr. 10
}

Ein vollstảndiger oder gekürzter Abdruck der Referate ist ohne Genehmigung der Redaktion und des Verlages verboten!

Aas, H. 1852

Abaev, 1,0188 Abbott Laboratories 1439 , 1464, 1496 Abdinov, D. S. 0154 Abel, E. W. 0800 Abraham, F. F. 0652 Abraham, R. J. 0935 Abramams, S. E. D. 0160

Achvlediani, R. N. 1903

Achvlediani, z. G.

Adams, M. J. 1802

Adams, w. B. 1967

Adloff., J.-P. 07

Aerojet-General

Corp. 1894, 1970

Agaev, A. I. 0265

Agaev, J. 0415

Agaev, U. C. 1072 .

Ageev, E. P. 0975

Ageev, P. J. 0385

Ageeva, L. E. 0188
Aglickaja, K. V.

Agliulov, N. C. 1646

Agripat Soc. An.

Ahmad, I. 0016

Airoldi, G. 0445

Aitova, E. F. 1198

Aj1, S. J. 1276

Akashi, N. 0631

A B B Bofors 1494

Aladzalova, N. A.

0627

Alamichel, C. 0876 ,

0884,0885 C. 0876,

Alberty, W. J. 0978

Albrecht, P. 1289

Albright \& Wilson

(Australia) Pty.

Aldrich, R. G. 0646

Alei jun., M. 0083

Aleksanjan, V. T.

0035
Alekseev, N. V. 0121

1583 , N. V. 0121,

Aleskovskij, v. B.

0676

Alexander, H. 0210

Alfenaar, M. 0589

Ali, S. S. 1239

Allidzanov, M. A.

Aliev, D. A. 1765

Aliev, G. M. 0154

Aliev, M. I. 0412

Alla, M. 0912

Allanazarov, A, 0405

Allen Jun., D. S. 136

Allen, M. J, 1450

Allied Ch, $\mathrm{s}$.

Corp. 1806, 1823, 1848,

Corp. 1806, 1823, 1848. Babaev, I. K. 0078
1924. 1957, 2022, 2071 Babak. K. A. 0605

Allison, K. 1941
Alt, L. J. 0358

Altenburg, K. 0826

Alter, A. 1464

Alti. G. De 0496
Altsuller, v. M. 0182

Altsuller, V. M.

Altuch

1949
American Cyanamid

Co. 1363, 1364, 1365

1359. 1396, 1488, 1508

merican Home

Products Corp.

Products Corp.

American Metal

Climax, Inc., 1839

Amphi $J 210$

Anand, M. L, 786

Anantaraman, $\mathbf{R}$.

1076

Anaskin, M. G. 0122

Anbar, M. 0736

Anderson, G. H. 1104, Ballman, A. A. 0463 Balluffi, R. W. 0233

Benoit, R. L. 0552

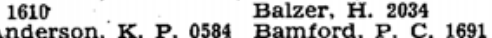
Anderson, P. P. 0584 Bamford, P. C. 0471 Ban, G. du 1193

Andrade, C. 027i ${ }^{2471}$ Ban, G. 1u 1191

Andraud, M. 1020 Banciu, M. 1101

Andreev, G. A. 0296 Banerjee, R. L. 0258

Andreeva, E. J. 0077 Ban-ya, S. 0529

ndreeva, E. P. 0322, Bansal, R. C. 0672

ndreu, P. 1093, 1094 Baptista, A. 0948

Andrew, J. E. 0495 Baranauckas, C. F

$\begin{array}{ll}\text { Andrews, F. C. } 0500 & 1928 \\ \text { Andrews, L. J. } 1073 & \text { Baranauckas, C. F. }\end{array}$

Andrzejczak, J. 0687

2010 .

Angiolini, J. 2025 Baranowski, B. 0530

Anikin, A. G. 0152 Baratange, J.-P. 1020

Anisimova, G. F. 1678 Barbenza, G. H.

Barca-Galateanu, D.

Antonenko, T. I. 05020905

Antonov, v. N. 1874 Barco, A. 1165

0358 Barer, J. A. 0501

Apers, D. J. 0749 Barnes, R. F. 001

Barnett, R. 1113

Baroncelli, F. 1138

Arbuzov, M. P. 0223

Archbold, R. O. 1670

1473

Aref'ev, A. V. 1524

Arenas, B. 1094

Argenziano, T. 2049

Arizmendi, L. 0709

Armbrust, H. 2057

14

Benzon, J. V. 1709

Benzing, E. P. 1897

Beran, M. 0560

Beranac, J. 0840

Berberich, R. 1738

Berends, w. 2051

1004

Berg, A. 2032

Berg, H. 1041

Berge, D. D. 1209

Berge, P. 0299

Berger, L. I. 0436

Bergerhoff, G. ${ }^{0245}$

Bergman, I. 1627

Bergstein, A. 0533

Bernard, M. L. 063

Bernardi, R. 1074

Bernstein, S. 1396

Berry, C. R. 0173

Berther, C. 2008

Berthon, G. 0637

Bertocchi, G. 0553

Bertoli, M. A. 1267

Barrall II, E. M. 0936 .

0940 . P. 0923

Bartels-Keith, J. R.

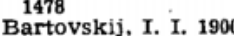

Bartura $J .0684$

Barzdajan, P. P. 012

Basa, S. J. 017i

Bassett, W. A. 1582

Basu, N. K 129

Arndt, Y. 1632 1132 Bate, I. C. 1594

Arnell. R. D. 1581 Batley, M. 1019

Arnikar, H. J. 1620

Arnold, A. J. 152

Arnold, R. 0689

Arrington jun., J. S.

1578

Artamonov, O. M.

Asada, E. 0947

Ascher, E. 0469 0243, $\begin{aligned} & \text { Becconsall, J. K. } 0106 \\ & \text { Becker, M. } 2000\end{aligned}$

Beckstead, M. W.

0694

Battisti, 1112 .

Bauer, S. H. 0929

Baukov, G. N. 1890

Baum, E. Do03 11

Baumann, H. 2081

Bayer, O. 2096

Bertrand, J. 2092

Bespalko, O. P. 0613

Betrencourt-Stirne-

Bettinali, C. 0347, 035

Bettoli, M. G. 0553

Betts, J. 1067

Bever, M. B. 0274

Bevill, R. D: 1711

Bezukladnikov, A. B.

Bezúkov, G. N. 0155

Biarnais, P. 1991

Bibas, I. 0510

Biel, J. H. 1463

Bienvenuee, A. 0002

Biggar, G. MCL. 1817
Bigorgne, M. 0069

er, D. 0780

Billiet, Y. 0192, 0778

Ltd, 1354

Birbeck, F. E. 0171

Birch, C. G. 1662

Bishara, S. W. 168

Bissara, S. W. 1682

Bittrich, H.-J. 0964,

0984

Aednjagina, N. P

Atherton, N. M. 0821

Bedrosian, A. J. 163

Atlas Chemical
Industries, Inc. 1948

Atoda, T. 0535

Atoda, T. 0535 $\quad$ Behrens, H. 0003

1516 . 1516

University 1446

Be

vakjan, N. O. 1673 Bejles, R. G. 0789 ,

Aven, M. 0428, $0433 \quad 0792$

Avidon, V. V. 1203

Azam, N. 0293

Azman, A, 0038

Azou, P. 0539

Babenko, G. A. 1272 Beljaev, A. D. 0268

Babeskin, A. M. 0745 Beljaev, V. D. 0186

Babko, A. K. 1631 Beljaeva, A. I. 032

Babuskina, T. A. 0240 Bell, S. C. 1386

Back. R. A. 0141, 0731 Bell, T. N. 1043

Badalov, M. F. 0412 Bell, W. O. 1984

Bader. R. F. W. 0026 Bellay, M. G du 185

Badische Anilin \& Bellettini, A. G. 1186

Soda-Fabrik AG Belous, M. V. 0444

2019, 2030, 2042, 2057, Belova, L. N. 1968

. 2079, 2080, 2081, Benarroche M. 0857

2094, 2095 20144

Bailes 1 . 1013, 1014 Bender, M. 0992

Bailey, P. S. 1990

Bendz, G. 1299

Benesor 0252

Bajdakov, L. A. 0439

0429

aaker, J. H. 1730

N. 066

Balfour, W. J. 0869
Bengtzelius, A. 028

Ben-Naim, A. 0600

Bennett, J. E, 0367,

0368 . 0639

Boldyrev, B. G. 1916

Bolitin, B. M. 1966

Boltz, D. F. 1660

Bombieri, G. 0249 , 0250

Bon, W. F. 1722

Bonc-Ósmolovskij,

A. G. 1529

Bonczyk, P. A. 0058

Bond, G. C. 0695

Bondarenko, E. G.

Bondarev, I. F. 0671

Bonnett, R. 1189

Bonnier, J. M. 0966.

Bonora, P. L. 0697

Bordwell, F. G. 1102

Borecky, J. 177

Brisova, Z. U. 0427,

Borrell, P. 1129

Borom, M. P. 1760

Boruch, M. 170339

Boschi, T. 0812

Boseell, G. G. J. 000

Bossard, W. 2099

Bouchaud, J.-P. 0483

Bouche, R. 1314

Boule, P. 0844, 1555

Bowers, A. 1408

Bozman, A. W. R. 0047 Bozzini, S. 1214

Braconaro, F. J. 1307

Bradley, K. H. 1696

Bradley, M. P. T. 168

Bradshaw, J. S. 1185

Braggio, F. 0520 C. L.

1322

Brand, E. 2039

Brand, J. C. D. 0853

Brandstetter, A. 0264

Brandt, R. 1241

Brannan, J. R. 0575

Bratu, C. 1084

Braun, W. 2094 


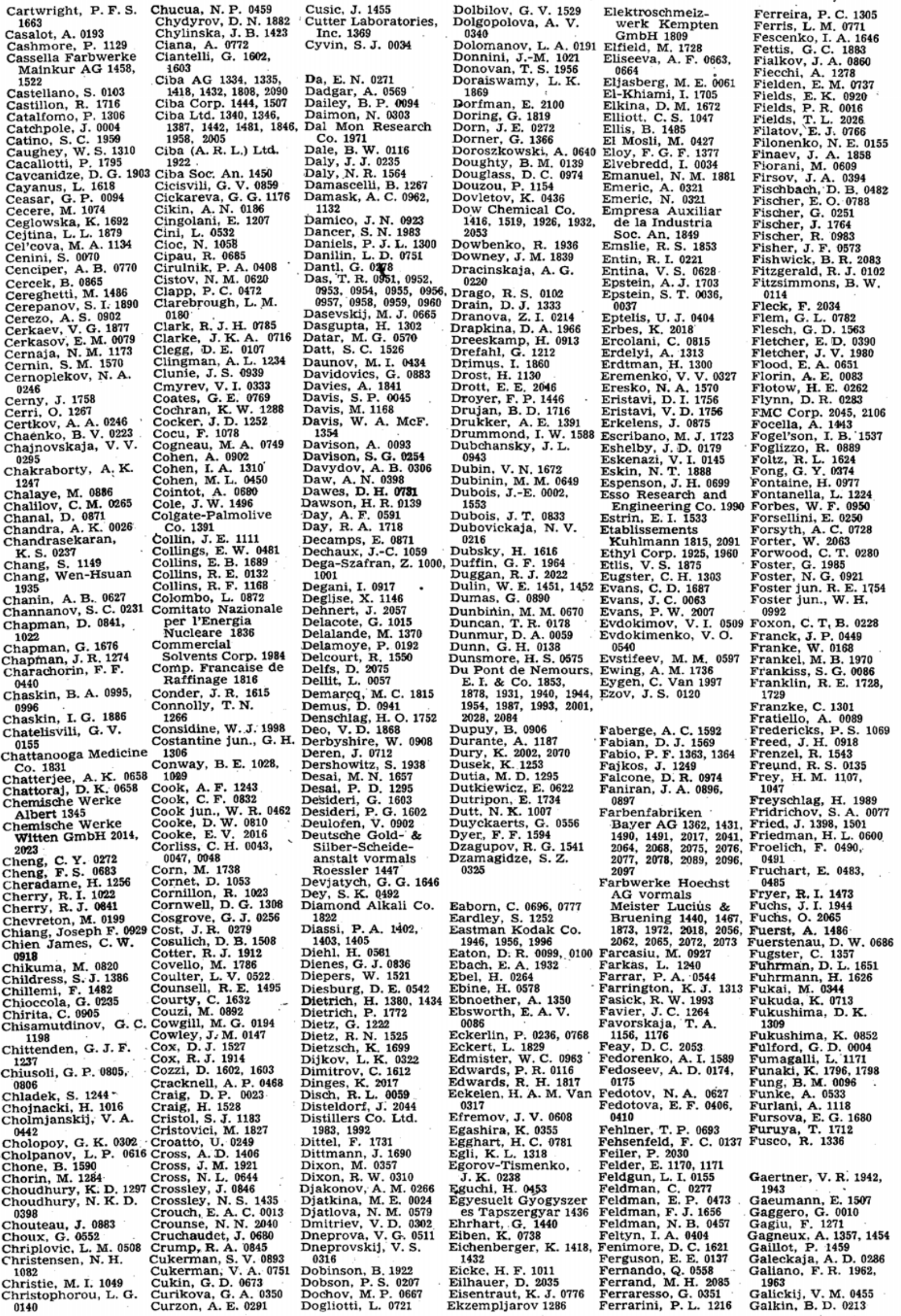




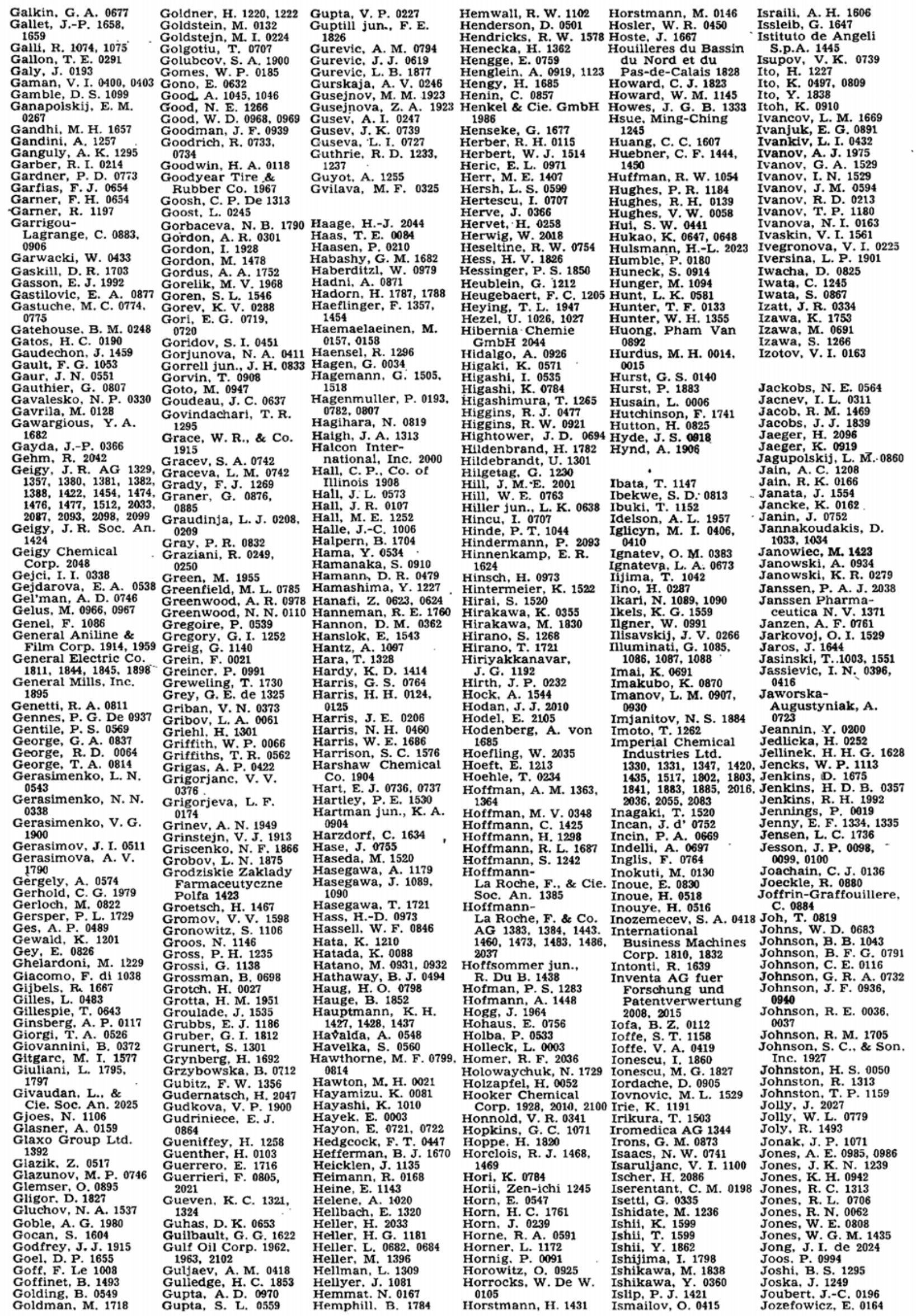




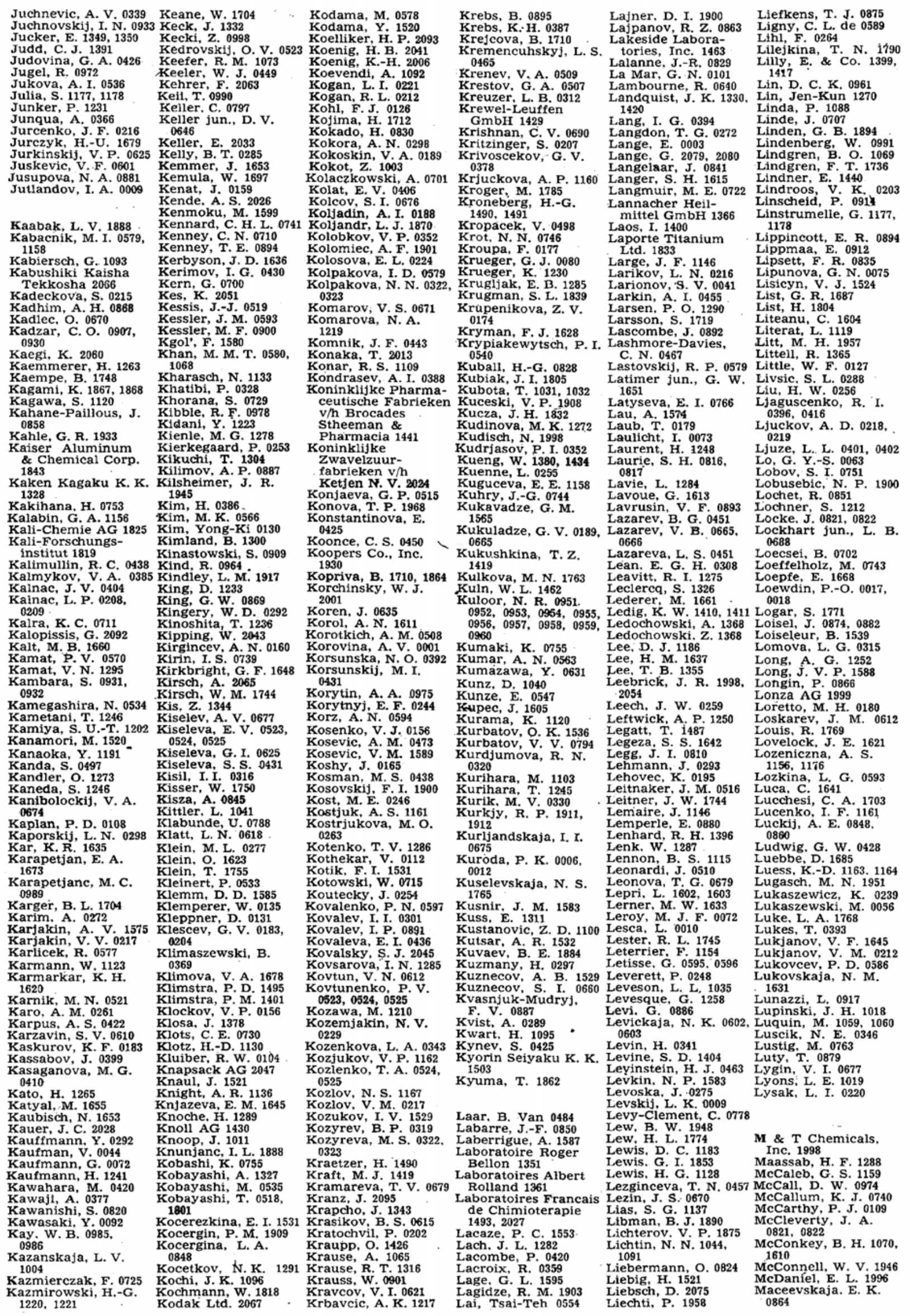




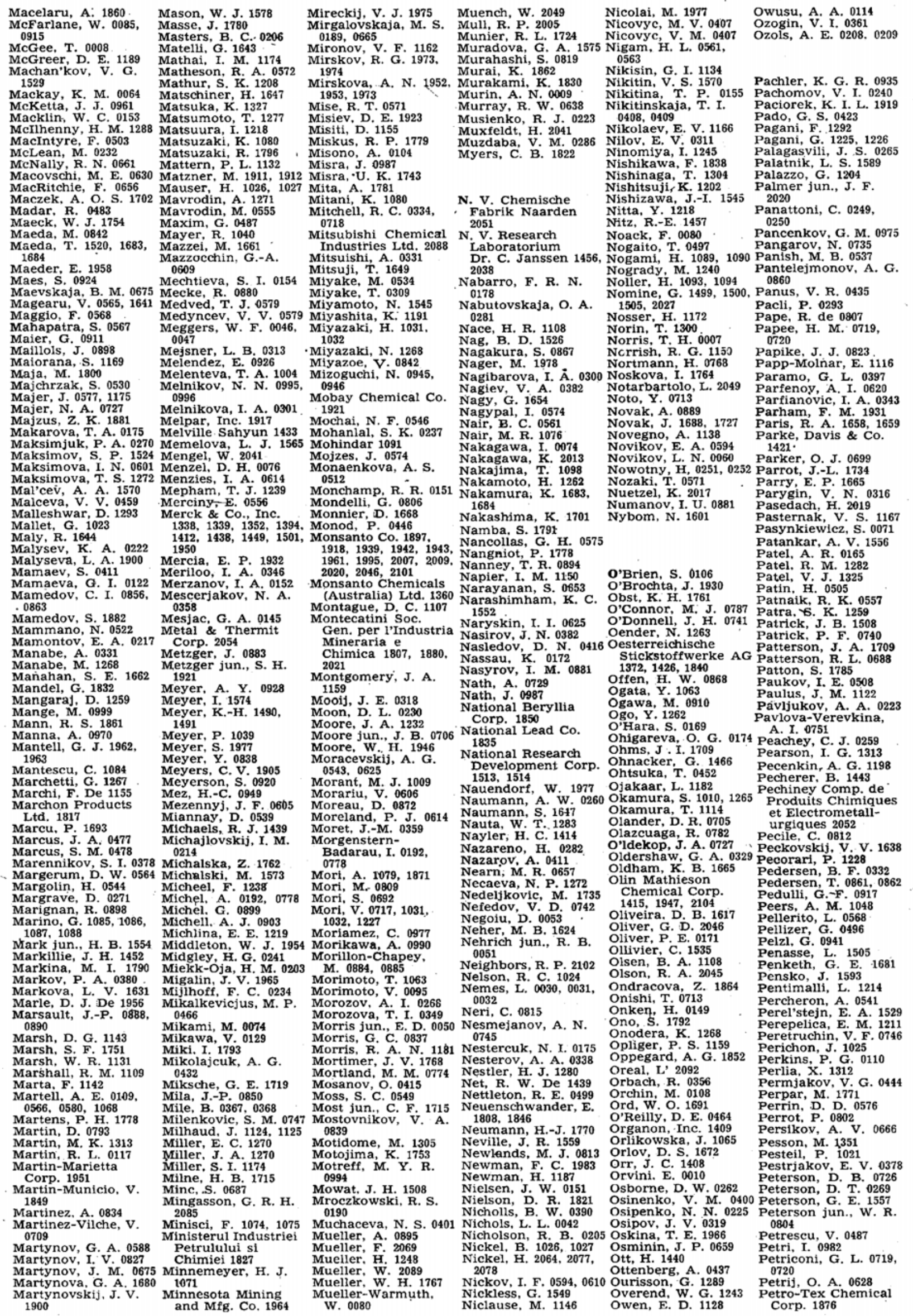




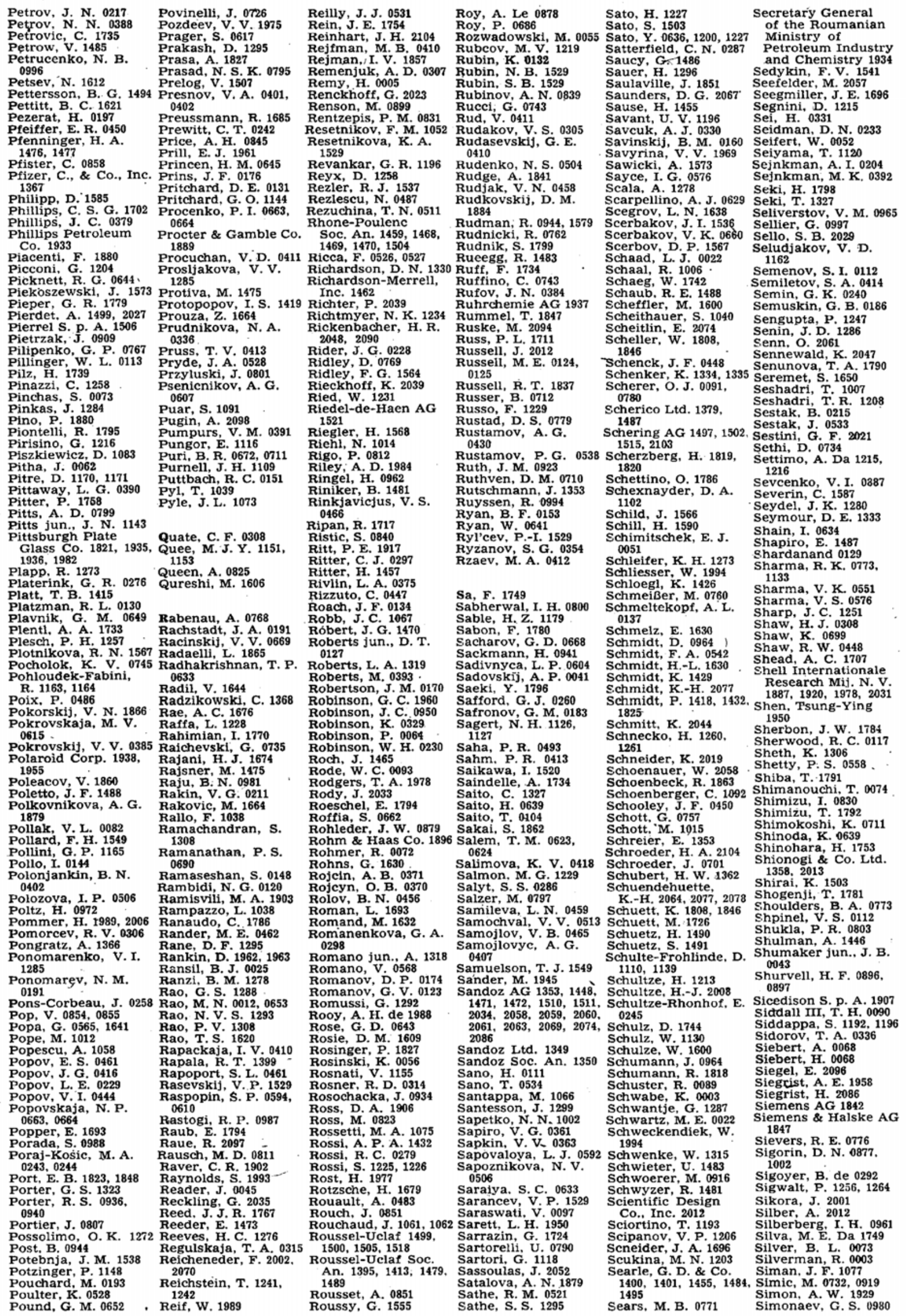




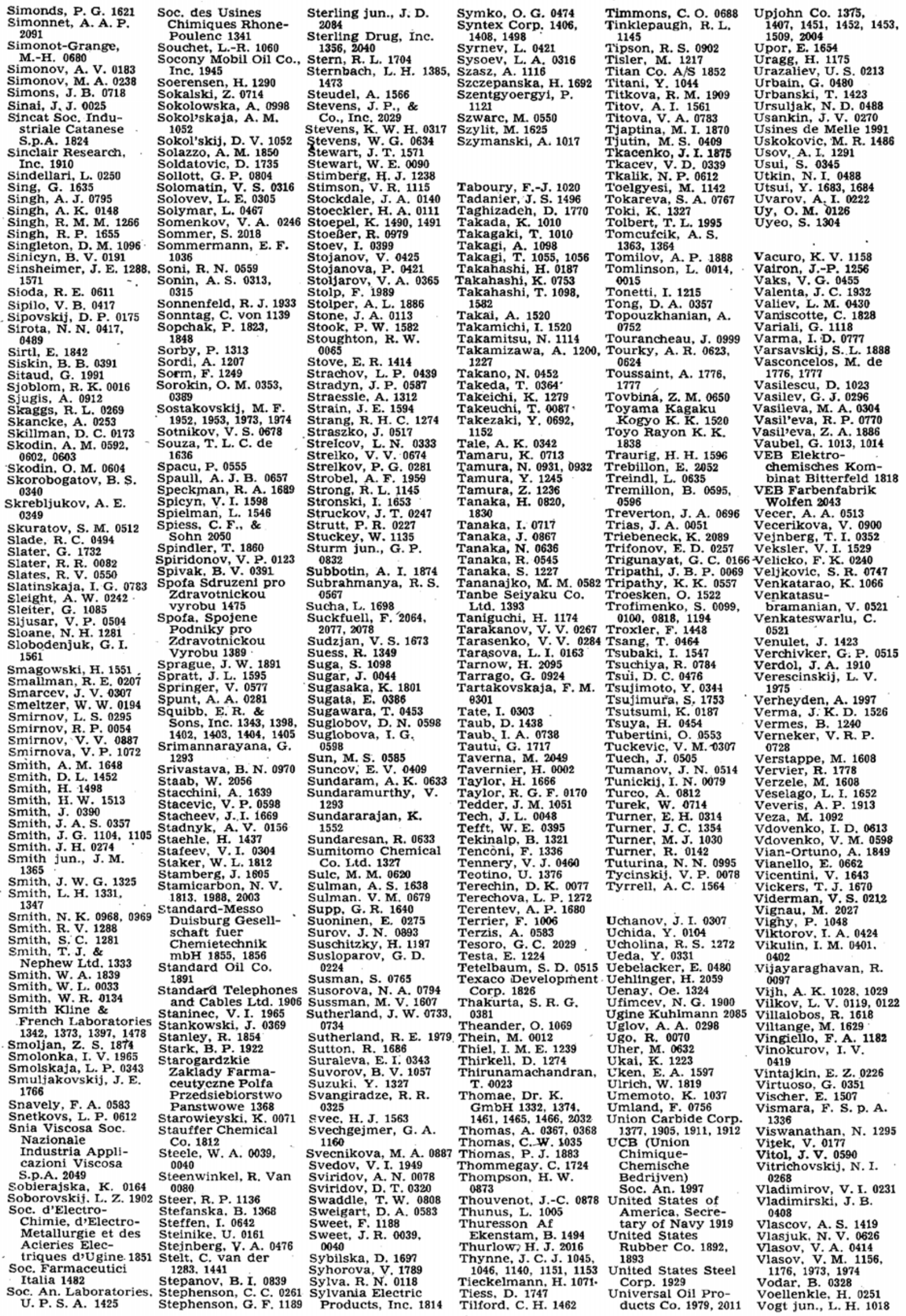




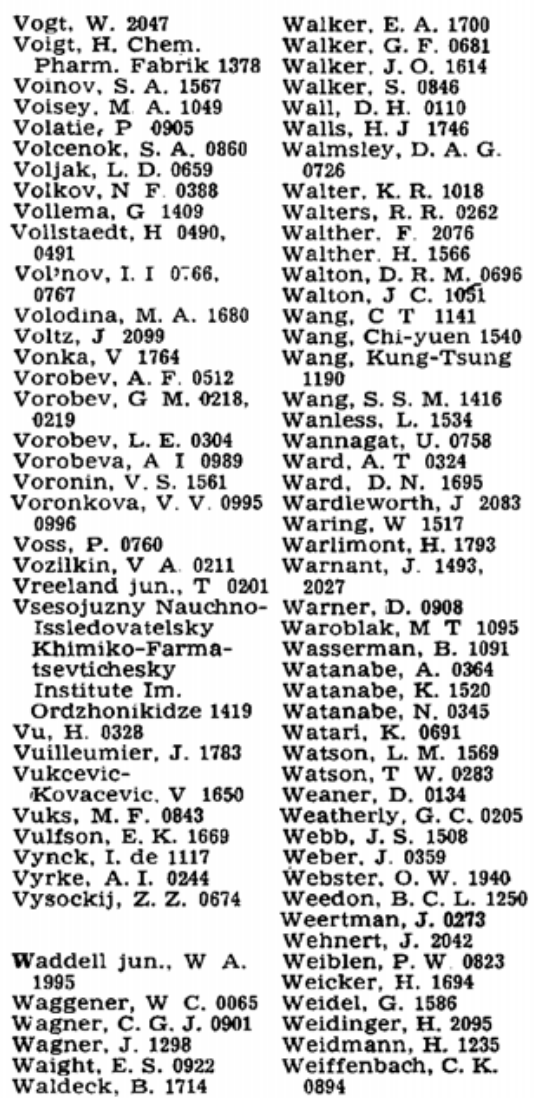

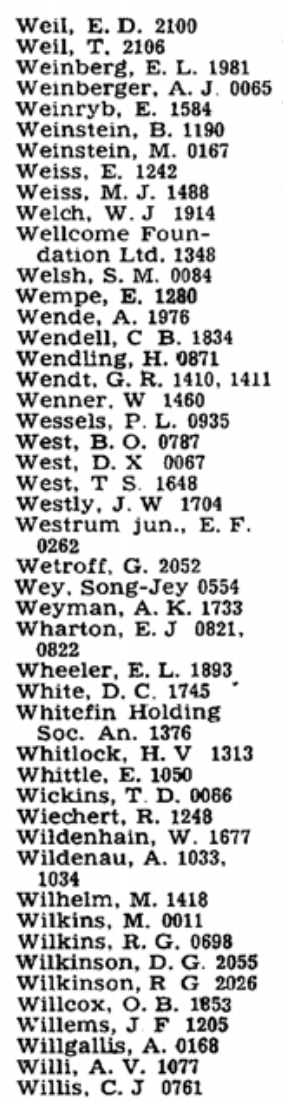

Willis, J. S. 0448 Williams, D. R. 0853 Williams, G. 0847 Williams, J. C. 1733
Williams, R. J P. 011 Williams, R. J P. 0116 Williams, R. P 1508 Williamson, J. E. Wills, R. D. 1736
Wilsdorf, H. G. F 0176 Wilson, C G 0290 Wilson, C G 0290 Wilson jun.. E. B.

Wilson, W. E. 0042

Wint, M. H. 1929 Winget, R. L. 1232 Winkelm. D. 1266 Winscom, C. J. 0821 Winston, A, 1251 Winstrom, L. O. 2022 Winter, E. 1568 Winter. W. 1266 Winterfeld, K. 1294 2079, 2080 wirtz, S. 143 Wiswall, R. H. 0531 Witt, A. F 0190 Wittmann, H. 1175 Wodzinski, R. J. 141 Wojtczak, J. 0723. Wojtkowiak, B. 0997 Wojtkowiak, Wolf, F. 1829 Wolf, H C. 09 Wolf. T. 1609 Wolski, w 0369 Wood, C. J. 0731 Wood, C. J. ${ }_{2025}$ Woodhouse, E. J 0007 Wooley, E. M. 0584 Wright, E. H. M. 0993 Wright, J. 1453 Wright, J. B. 1452 Wright, J R. 1451 Wright, R D. 1446 Wronski, M. 1737 wygant, J C. 1961 Wyss. E. 1232
Yagi, H. 1246 Yagi, S. 0518 Yamada, F 0910 Yamada, K. 1277 Yamaguchi, G. 1792 Yamamoto, O. 008 Yamamoto, S. 1245 Yamamoto, Y 0809 Yanagida, H 0708, 1792

Yanal, M. 1796 Yannoni, C. S. 009 Yano, T. 1277 Yasuda, K. 1701 Yasuda, K. 1713 Yasuoka, Y. 0377 Yato, Y. 0753 Yeh, H. C. 0661 Yeh, H. C. 0661 Yennie, D R. 0027 Yeranos, W. A. 0028,

Yoda, N 1103 Yokoyama, A. 0820 Yokoyama, A. 18 Yoneda, H. 0095 . 0809

Yonemitsu, O. 119 Yonemitsu, O. 1191 Yoshida, T. 1740 Yoshikawa, M. 159 Yoshinaga, H. 0331 Yosida, K. 0470 Young, H. S. 1996 Young, R. P. 0591 Yu, N'T 0591 uasa, K. 1279 Yukawa, Y. 114?

Zabotinskij, M. E. 0376 Zacharov, V. A. 1541 Zadumkin, S. N. 0667 Zahn, U. 0750 1881 Zajdman, G Ij 0400
Zajdman, S. A. 0400 Zana, R. 0976 Zanin, S. 1720 Zargarova, M. I. 0538 Zarinova, T. N. 1968 Zarli, B. 0249 Zarubina, O. A. 0263 Zaslow, B. 0943 Zaugg, H. E. 1439
Zavoianu, D. 1078 , 1079. 1871 Zec. J 1709 Zeeck, A. 1287 Zeile, K. 1427, 1428 , Zejnalov. A. A. 0382 Zelova. V. S. 0678 Zeludev, I. S. 0457 Zemcuzin, S. G. 190 Zemljanov, M. G. 0246 Zemljanskij, N. I. 1173 Zemskov, G. V. 0212 Zerjukina, L. 1966 Zerjukina, L. S. 1966 Zerweck, w 1522 Ziemelis, K. M. 0864 Zilberstejn, C I. 1642 Zimmermann, E. 1542 Zimmerman, H. K.

Zingales, F. 0790 Zinnsstag. C. 1999 Zirm, K. L. 1366 Zissis, E. 1234 Zlatkis, A. 1621 Zotov, S. B. 0887 Zuercher, K. 17871788 Zuev, V A. 0488 Zuev, $V$ M. 0265 Zukal, A. 0670 Zuman, P 1064 Zungletu, G I. 1211 Zuravlev, S. V 1166 , 1969 Zuravlev, V. A. 0543 Zyl B. Van 0138 Zwolinski, B. J. 0852

Patentregister

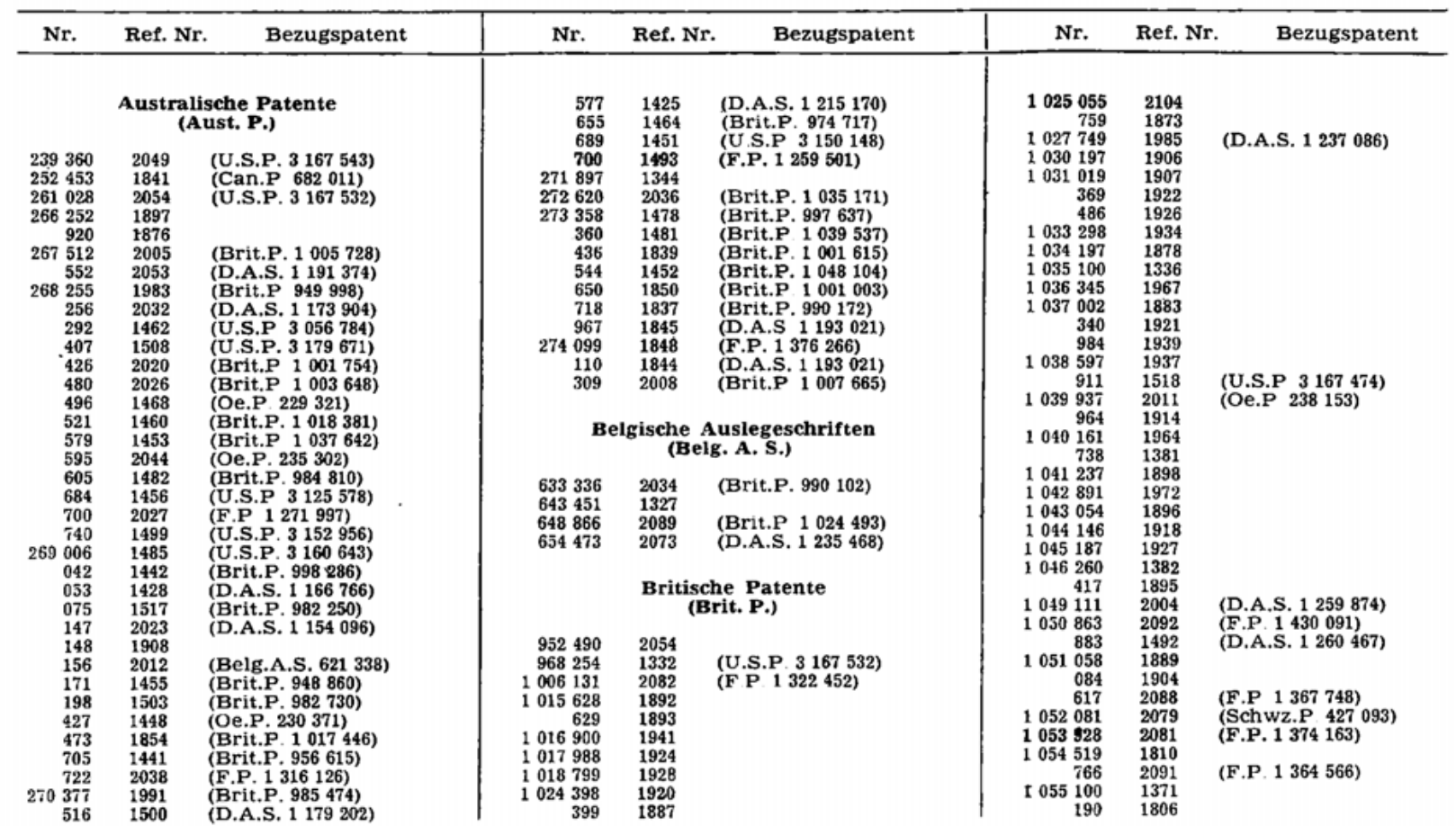

